\title{
Alltagsintegrierte Förderung der Schriftsprache im Vorschulalter
}

\section{Susanne Geyer, Andreas Hartinger \& Gisela Kammermeyer}

Die phonologische Bewusstheit gilt als ein bedeutsamer Prädiktor des Erfolgs beim Lesen- und Schreiben lernen, der sich bereits vor Schuleintritt erfolgreich fördern lässt (z.B. Küspert \& Schneider 2008). Dazu existierende Forschungsbefunde beziehen sich hauptsächlich auf die Wirkung additiver Trainingsprogramme, in denen Vorschulkinder in einer separaten Kleingruppe gefördert werden. Unklar ist, wie ein Förderansatz wirkt, bei dem Erzieherinnen im Kindergartenalltag in der Regelgruppe bereichsspezifische Kompetenzen im Schriftspracherwerb anregen im Sinne einer entwicklungsorientierten Vorbereitung auf die spätere Bildungslaufbahn des Kindes. In diesem Beitrag sollen Ergebnisse einer Längsschnittstudie ${ }^{1}$ im letzten Kindergartenjahr dargestellt werden.

\section{Schriftsprachliche Förderansätze im Kindergarten}

Trainingsprogramme zur phonologischen Bewusstheit erfahren in deutschen Kindergärten breite Akzeptanz. Sie zeichnen sich aus durch einen zeitlich vorstrukturierten Trainingsplan mit klar vorgegebenen Förderinhalten und detaillierten methodischen Handlungsanweisungen. In Anlehnung an die Klassifikation nach Kluczniok, Roßbach und Große (2010) können sie zu den angebotsorientierten bereichsspezifischen Förderansätzen gezählt werden. Bei unausgelesenen Stichproben wirkt ein vorschulisches Training sowohl kurz- als auch langfristig positiv auf die kindliche Entwicklung der phonologischen Bewusstheit und die Schriftsprachkompetenzen in den ersten beiden Grundschuljahren (Lundberg, Frost \& Petersen 1988; Schneider, Roth, Küspert \& Ennemoser 1998). Eine Trainingsversion, die mündlich geübte Fertigkeiten der phonologischen Bewusstheit mit der Förderung von Graphem-Phonem-Korrespondenzen kombiniert, erzielt dabei stabilere Langzeiteffekte als die Förderung einer einzelnen Komponente (Hatcher, Hulme \& Ellis 1994; Schneider, Roth \& Ennemoser 2000).

In alltagsintegrierten Förderansätzen werden Lernprozesse mit der persönlichen Erfahrungswelt der Kinder verknüpft. Ausgehend von Alltagsanlässen, von ihren Spielsituationen oder von selbst gewählten Projekten werden kindliche Kompetenzen unter hoher Eigenaktivität des Kindes bezogen auf diese Situation erweitert (Roßbach 2008). Ein Beispiel dafür aus dem Schriftspracherwerb ist das sogenannte „Literacy-Center“. Dabei handelt es sich um eine Spielund Lernumgebung, welche mit Materialien angereichert ist und die Kinder zu Lese- und Schreibaktivitäten herausfordert (Kammermeyer 2010). Befunde aus der amerikanischen Play and Literacy-Forschung (Morrow \& Rand 1991; Christie \& Roskos 2006) zeigen, dass Kinder vermehrt Lese- und Schreibaktivitäten in ihr (Rollen-)Spiel einbauen, wenn sie solche schreib/lesebezogenen Materialien angeboten bekommen. Diese Spielmaterialien können allgemein und/oder themenbezogen sein, vor allem aber sollten sie kindgemäß, authentisch und nützlich sein. Darüber hinaus verbessern Vorschulkinder durch das Einrichten von Rollenspielecken signifikant ihr Verständnis über die Funktion von Schrift, und ihr Spiel verändert sich qualitativ.

\footnotetext{
${ }^{1}$ Die Studie wurde vom Bundesministerium für Bildung und Forschung (BMBF) unter dem Förderkennzeichen 01 JG0915 finanziell unterstützt.
} 


\section{Forschungsdesiderat und Fragestellung}

Bisher wurden Kinder einer Vergleichsgruppe, die keine spezifisch schriftsprachliche Förderung im Kindergartenalltag erfuhren, mit Kindern verglichen, die eine zusätzliche schriftsprachliche Intervention über einen definierten Zeitraum erhielten. Unklar ist jedoch, wie eine alltagsintegrierte schriftsprachliche Intervention im Vergleich zu einem Trainingsprogramm der phonologischen Bewusstheit wirkt. Aufbauend auf den theoretischen Vorüberlegungen soll daher folgende Fragestellung untersucht werden: Unterscheiden sich Vorschulkinder in ihrer schriftsprachlichen Leistungsentwicklung in einem alltagsintegrierten Literacy-Setting im Vergleich zu einem angebotsorientierten Trainingsprogramm?

\section{Methodik der Untersuchung}

Dieser Frage wurde in einem quasi-experimentellen Prä-Post-Kontrollgruppendesign nachgegangen. Dabei wurden schriftsprachliche Vorläuferfähigkeiten ein Jahr vor der Schule $\left(\mathrm{t}_{1}\right)$ erfasst und nochmals am Ende des letzten Kindergartenjahres $\left(\mathrm{t}_{2}\right)$.

Als theoretische Grundlage für die Entwicklung eines alltagsintegrierten Literacy-Treatments diente das Konzept des „Literacy-Centers“. Schwerpunktmäßig erhielten Erzieherinnen den Auftrag über neun Monate lang schriftsprachliche Aktivitäten in Alltagssituationen oder Projekten mit der ganzen Kindergartengruppe aufzugreifen, im Rollenspiel zu intensivieren und mit Spielen zur phonologischen Bewusstheit anzureichern. Als Vergleichsgruppe wurde eine trainierte Kontrollgruppe gewählt. Diese arbeitete bereichsspezifisch angebotsorientiert und griff täglich zehn bis 15 Minuten über 20 Wochen verteilt in altershomogenen Kleingruppen verschiedene Lausch-, Reim-, Silbenspiele sowie Übungen zur Lautanalyse aus dem Würzburger Trainingsprogramm 1 (Küspert \& Schneider 2008) auf. Zu beiden Förderansätzen (Literacy-Center und Würzburger Trainingsprogramm 1) erhielten die Erzieherinnen vorab eine dreitägige Fortbildung.

An der Studie nahmen 229 Vorschulkinder aus 14 Kindergärten der Region Augsburg teil. Für die Auswahl wurden interessierte Kindergärten nach relevanten Kriterien gematcht (wie z.B. Anzahl der Vorschulkinder, regionaler Einzugsbereich, Vorwissen der Erzieherinnen) und den Untersuchungsbedingungen zugewiesen. Sieben dieser Kindergärten lagen in städtischen, die restlichen sieben in ländlichen Gebieten. Die Gesamtstichprobe bestand aus 48,5 \% Mädchen und 11,7 \% Kindern mit nicht-deutscher Familiensprache. Zu Beginn der Untersuchung betrug das durchschnittliche Alter der Kinder 5,5 Jahre (SD = 4 Monate). Die Stichprobe verteilte sich wie folgt: Am Literacy-Treatment nahmen 185 Vorschulkinder aus 30 Kindergartengruppen in elf Einrichtungen teil, das Würzburger Trainingsprogramm wurde von 44 Vorschulkindern in acht Kindergartengruppen aus drei Einrichtungen durchgeführt.

Als abhängige Variable wurde die phonologische Bewusstheit im engeren Sinn spezifiziert. Erhoben wurden mit dem adaptiven Testinstrument „wortgewandt und zahlenstark“ (Moser \& Berweger 2007) Aufgaben zur Lautanalyse, -segmentation und -manipulation (41 Items; $\mathrm{t}_{1}: \alpha$ $=.93 ; \mathrm{t}_{2}: \alpha=.93$ ). Zusätzlich wurde einmalig zu Untersuchungsbeginn die nonverbale, kognitive Leistungsfähigkeit mit Hilfe des CFT 1 von Weiß und Osterland (1997) kontrolliert (36 Items; $\left.\mathrm{t}_{1}: \alpha=.80\right)$. 
Um Unterschiede zwischen den Untersuchungsgruppen festzustellen, wurden hierarchische Regressionsanalysen (Geiser 2010, 201 ff.) mit Mplus gerechnet. Damit ist es möglich, die Kindergartengruppenzugehörigkeit zu berücksichtigen. Mit Intraklassenkoeffizienten (ICC) um .05 können wir davon ausgehen, dass bis zu 5 \% der Gesamtvarianz der abhängigen Va-riable ,phonologische Bewusstheit eng “ auf Unterschiede zwischen den einzelnen Kindergartengruppen ( $\mathrm{n}=38)$ zurückgeht. Nach Hox (2010) liegt demnach hinreichend Variation zur Durchführung einer Mehrebenenanalyse vor. In verschiedenen Regresssionsmodellen wurde sukzessive der Einfluss des Vortestwissens (Modell 1) und der Intelligenz (Modell 2) auf Individualebene und der Einfluss des Treatments (Modell 3) auf Kindergartengruppenebene getestet.

\section{Ergebnisse}

Tabelle 1: Mittelwerte (M) und Standardabweichungen (SD) der phonologischen Bewusstheit im engeren Sinn zum ersten und zweiten Messzeitpunkt für beide Interventionsbedingungen

\begin{tabular}{lllll}
\hline & \multicolumn{2}{l}{ Literacy-Treatment $(n=185)$} & \multicolumn{2}{l}{ Würzburger Training $(n=44)$} \\
\hline & $\mathrm{M}$ & $\mathrm{SD}$ & $\mathrm{M}$ & $\mathrm{SD}$ \\
\hline $\mathrm{t}_{1}$ & .376 & .214 & .394 & .226 \\
\hline $\mathrm{t}_{2}$ & .600 & .231 & .572 & .247 \\
\hline
\end{tabular}

Anmerkungen: $\mathrm{n}=$ Anzahl der Vorschulkinder; $\mathrm{t}_{1}=$ erster Messzeitpunkt; $\mathrm{t}_{2}=$ zweiter Messzeitpunkt.

Die beiden Untersuchungsgruppen unterscheiden sich zu Beginn des letzten Kindergartenjahres $\left(\mathrm{t}_{1}\right)$ nicht überzufällig in ihren Vorkenntnissen zur phonologischen Bewusstheit im engeren Sinn: $F(1,227)=0.22 ; \mathrm{p}=.635$. Die bivariaten Korrelationen zwischen der phonologischen Bewusstheit vom ersten zum zweiten Messzeitpunkt betragen im Literacy-Treatment $.79 * * *$ und in der Würzburger Trainingsgruppe .86*** und zeugen von einer hohen interpersonellen Stabilität.

Tabelle 2: Einfluss verschiedener Variablen auf den Lerngewinn in der phonologischen Bewusstheit im engeren Sinn

\begin{tabular}{llll}
\hline & Modell 1 & Modell 2 & Modell 3 \\
\hline Vorwissen pb eng & $.800^{* * *}$ & $.780^{* * *}$ & $.827 * * *$ \\
\hline Intelligenz & & .040 & \\
\hline Treatment & & & $.334^{*}$ \\
\hline $\mathrm{R}^{2}$ (Level 1) & $0.640^{* * *}$ & $0.641^{* * *}$ & $0.684 * *$ \\
\hline
\end{tabular}

Anmerkungen: pb eng = phonologische Bewusstheit im engeren Sinn; Literacy-Center(1), Trainingsprogramm $(0) ; * p<.05 ; * * * p<.001$; angegeben sind die standardisierten Beta-Koeffizienten (B) der hierarchischen Regressionsanalysen.

Am Ende des letzten Kindergartenjahres ( $\left.\mathrm{t}_{2}\right)$ zeigt sich, dass die Kinder der Literacy-TreatmentGruppe durchschnittlich mehr richtige Antworten im Bereich der phonologischen Bewusstheit im engeren Sinn lösten als in der Würzburger Trainingsgruppe. Der obige Befund zur hohen Bedeutung des Vortestwissens wird dadurch bestätigt $(p<.01)$ und klärt allein 64\% der Varianz auf Individualebene auf. Der Einfluss der Intelligenz auf den Lernzuwachs in der phonologischen Bewusstheit geht unter Berücksichtigung des Vorwissens verloren. Der Einfluss des Literacy-Treatments wird, unter Kontrolle der Vortestwerte in der phonologischen Bewusstheit im engeren Sinn, überzufällig ( $p=.021)$. 


\section{Diskussion und Ausblick}

Bezüglich des Lerngewinns in der phonologischen Bewusstheit im engeren Sinn ergeben sich keine Hinweise darauf, dass ein Trainingsprogramm zur phonologischen Bewusstheit wirksamer ist als die alltagsintegrierte Literacy-Förderung. Dieser Befund fügt sich ein in Erkenntnisse zum impliziten und expliziten Spracherwerb, wonach sowohl alltagsbezogene situationsorientierte Sprachanlässe lernförderlich sein können, als auch spezifische Lernsituationen für Kinder mit diagnostiziertem Förderbedarf (Knapp, Kucharz \& Gasteiger-Klicpera 2010). Wie aus der didaktischen Diskussion um Sprachunterricht hervorgeht, spricht für eine Förderung im Kindergartenalltag, dass Kinder nicht altershomogen gruppiert werden (z.B. nach Vorschulkindern), sondern von und miteinander in ihrer Peergroup lernen können. Nachteil ist, dass diese Art der Sprachförderung oft unsystematisch verläuft und Kinder mit Defiziten nicht rechtzeitig erkannt werden. Umgekehrt laufen gezielte, inszenierte Sprachfördersituationen Gefahr als „,verschult“ und „alltagsfremd“ von den Erzieherinnen in der Praxis umgesetzt zu werden. Demzufolge könnte es auch mit Blick auf die Förderung der Schriftsprache pädagogisch sinnvoll sein, alle Vorschulkinder alltagsintegriert, in ihrer natürlichen Umgebung auf die Schule vorzubereiten und isolierte Trainingsprogramme gezielt für Kinder mit Schwierigkeiten in der phonologischen Bewusstheit anzubieten. Allerdings sollte dieser erste Befund noch vorsichtig interpretiert werden. Um zu validen Aussagen zu kommen, müssen im nächsten Schritt Prozessmerkmale zur Durchführung und Wirksamkeit der Intervention (z.B. schriftsprachliche Anregungsqualität der Erzieherinnen) mit dem Lerngewinn der Kinder abgeglichen werden.

\section{Literatur}

Christie, J. F. (Hrsg.) (1991): Play and Early Literacy Development. Albany: State Univ. of New York Press

Christie, J. F. \& Roskos, K. (2006): Standards, Science, and the Role of Play in Early Literacy Education. In: Singer et. al. (2006): 57-73

Diller, A., Leu, H. R. \& Rauschenbach T. (Hrsg.) (2010): Wie viel Schule verträgt der Kindergarten? Annäherung zweier Lernwelten. München: Deutsches Jugendinstitut

Geiser, C. (2010): Datenanalyse mit Mplus. Eine anwendungsorientierte Einführung. Wiesbaden: VS Verlag für Sozialwissenschaften

Hatcher, P., Hulme, C. \& Ellis, A. W. (1994): Ameliorating early reading failure by integrating the teaching of reading and phonological skills: The phonological linkage hypothesis. In: Child Development 65. 41-57

Hox, J. J. (2010): Multilevel Analysis. Techniques and Applications. New York: Routledge

Kammermeyer, G. (2010). Förderung des frühen Schriftspracherwerbs im Rollenspiel. In: Spiel et.al. (2010): 70-74

Kluczniok, K., Roßbach, H.-G. \& Große, C. (2010): Fördermöglichkeiten im Kindergarten. Ein Systematisierungsversuch. In: Diller et. al. (2010): 133-152

Knapp, W., Kucharz, D. \& Gasteiger-Klicpera, B. (2010): Sprache fördern im Kindergarten. Umsetzung wissenschaftlicher Erkenntnisse in die Praxis. Weinheim: Beltz

Küspert, P. \& Schneider, W. (2008): Hören, lauschen, lernen 1. Sprachspiele für Kinder im Vorschulalter. Würzburger Trainingsprogramm zur Vorbereitung auf den Erwerb der Schriftsprache. (6. Aufl.). Göttingen: Vandenhoeck \& Ruprecht 
Lundberg, I., Frost, J. \& Petersen, O. (1988): Effects on an extensive program for stimulating phonological awareness in preschool children. In: Reading Research Quarterly 23.263-284

Morrow, L. M. \& Rand, M. (1991): Preparing the classroom environment to promote literacy during play. In: Christie (1991): 141-165

Moser, U. \& Berweger, S. (2007): wortgewandt \& zahlenstark. Test zur Ermittlung des Lern- und Entwicklungsstands bei 4- bis 6-Jährigen. Zürich: Kantonaler Lehrmittelverlag

Otto, H.-U. (Hrsg.) (2008): Die andere Seite der Bildung. Zum Verhältnis von formellen und informellen Bildungsprozessen. Wiesbaden: VS Verlag für Sozialwissenschaften

Roßbach, H. G. (2008): Was und wie sollen Kinder im Kindergarten lernen? In: Otto (2008): 123-133

Schneider, W., Roth, E., Küspert, P. \& Ennemoser, M. (1998): Kurz- und langfristige Effekte eines Trainings der sprachlichen (phonologischen) Bewusstheit bei unterschiedlichen Leistungsgruppen: Befunde einer Sekundäranalyse. In: Zeitschrift für Entwicklungspsychologie und Pädagogische Psychologie 30 (1). 26-39

Schneider, W., Roth, E. \& Ennemoser, M. (2000): Training phonological skills and letter knowledge in children at risk for dyslexia: A comparison of three kindergarten intervention programs. In: The Journal of Educational Psychology 92 (2). 284-295

Singer, G. D., Golinkoff, R. M. \& Hirsh-Pasek, K. (Hrsg.) (2006): Play=Learning. How Play Motivates and Enhances Children's Cognitive and Social-Emotional Growth. New York: Oxford University Press

Spiel, C., Schober, B., Wagner, P. \& Reimann R. (Hrsg.) (2010): Bildungspsychologie. Göttingen: Hogrefe

Weiß, R. H. \& Osterland, J. (1997): CFT 1. Grundintelligenztest Skala 1. Frankfurt/Main: Pearson 